\title{
Constraints on using the dual-task methodology to specify the degree of central executive involvement in cognitive tasks
}

\author{
MARY HEGARTY \\ University of Califormia, Santa Barbara, California \\ PRITI SHAH \\ University of Memphis, Memphis, Tennessee \\ and \\ AKIRA MIYAKE \\ University of Colorado, Boulder, Colorado
}

\begin{abstract}
The dual-task paradigm has been used to examine the role of the central executive in various cognitive tasks. In these studies, performance decrements in primary cognitive tasks performed concurrently with secondary executive tasks have been interpreted as evidence for the involvement of the central executive in those primary tasks. In the present study, we examined the effects of different secondary tasks on performance of three psychometric visuospatial tasks. The decrement in performance of these tasks when they were paired with secondary executive tasks was smallest for the psychometric task considered to most heavily involve the central executive and largest for the task considered least demanding of executive mechanisms. We propose that, when applied to the assessment of central executive involvement, the prevalent simple dual-task logic does not always apply. Special conditions that limit the application of the dual-task methodology include two inherently related factors-a response selection bottleneck and a strategic tradeoff between primary and secondary tasks.
\end{abstract}

The dual-task paradigm has been widely used in working memory research. In particular, it has been used within the framework of Baddeley's (1992; Baddeley \& Hitch, 1974) influential model of working memory, which proposes that working memory consists of a central control structure called the central executive and two peripheral, domain-specific "slave" systems called the phonological loop and the visuospatial sketchpad. In the dual-task paradigm, a cognitive task (a primary task) is performed both by itself and concurrently with a secondary task considered to tap one of the subcomponents of working memory. If the secondary task disrupts performance of the primary task (relative to the condition in which the primary task is performed alone), it is inferred that the working memory subcomponent tapped by the secondary task is involved in the performance of the primary task. For example, repeatedly articulating a familiar word or phrase (such as, the, the, the) is an often used secondary task for the phonological loop component. This articulatory sup-

We thank Elizabeth Collin, Christina Lorenzen, and Shirley Wong for assistance with data collection and Satoru Saito for useful comments on an earlier draft of the article. This research was supported in part by Grant N00014-96-1-0525 from the Office of Naval Research to M.H. Correspondence regarding this article should be addressed to M. Hegarty, Department of Psychology, University of California, Santa Barbara, CA 93106 (e-mail: hegarty@psych.ucsb.edu). pression technique selectively disrupts performance on a host of verbally mediated tasks, such as immediate serial recall and mental arithmetic, which points to the involvement of the phonological loop in these tasks (Baddeley \& Logie, 1999; Gathercole \& Baddeley, 1993). Similarly, spatial tapping (i.e., tapping the four corners of a square in sequence) has often been used as a secondary task for the visuospatial sketchpad (particularly its spatial, as opposed to its visual aspects), and this impairs people's performances on various visuospatial tasks, such as mental rotation (Logie, 1995).

Recently, this dual-task paradigm has been applied in order to examine the role of the central executive in various complex cognitive tasks. The secondary task most widely used in this context is oral random generation (Baddeley, 1966), which requires participants to continuously generate a series of numbers or letters in as random an order as possible. This task is considered to tap the central executive because it requires actively monitoring candidate responses and suppressing responses that would lead to well learned sequences, such as $1-2-3-4$ or $a-b-c-d$ (Baddeley, 1996). The mental retrieval of candidate responses from the total number of possibilities might constitute an additional and independent source of central executive involvement (Towse, 1998). Several recent studies have shown that random generation interferes with participants' performance on a host of complex cognitive tasks, such as 
playing chess (Robbins et al., 1996), mental arithmetic (Logie, Gilhooly, \& Wynn, 1994), the Brooks Matrix task (Salway \& Logie, 1995), and syllogistic reasoning (Gilhooly, Logie, Wetherick, \& Wynn, 1993). A conclusion frequently drawn from such results is that these cognitive tasks involve the central executive component of working memory.

We have some concerns about the use of the dual-task paradigm to specify the degree of central executive involvement in complex cognitive tasks. In this study, we raise two closely related factors that need to be considered when one is applying the dual-task methodology to study the role of the central executive. We illustrate the importance of these factors by presenting a counterintuitive finding from a dual-task experiment, which is that concurrent performance of executive tasks can interfere more with a simple perceptual speed task than with a complex spatial reasoning task that seemingly demands more executive processing. The main goal of this study is to specify some conditions under which the simple dual-task logic might not hold and thereby to contribute to the sharpening of the dual-task methodology when it is applied to the central executive component.

According to the simple dual-task logic, any cognitive task that involves the operation of the central executive should be disrupted by concurrent performance of executive tasks, such as random number generation, even if there is no interference at the level of a common input modality or a common mode of response. Furthermore, this logic also suggests that the degree of performance disruption should correlate with the extent to which the target cognitive task involves the operations of the central executive.

There are a number of reasons why this straightforward logic might not apply under certain circumstances. One concerns the existence of a central bottleneck for response selection. Pashler (1994) has argued that a bottleneck can occur in dual-task situations at the response selection phase (i.e., two responses cannot be selected at the same time) even when other perceptual and motor processes can co-occur. Executive tasks like random number generation require rapid, constant response selection. Thus, it is possible that concurrent performance of executive tasks can severely interfere with the performance of a primary task that also involves rapid response selection but requires little involvement of the central executive (such as tasks that require sequences of simple perceptual judgments).

Another reason concerns a strategic tradeoff between primary and secondary task performance. Some studies have reported that regardless of which task is designated as primary, people tend to allocate more resources to the one that they perceive to be more demanding (see, e.g., Bourke, Duncan, \& Nimmo-Smith, 1996; Navon \& Gopher, 1979). This type of strategic tradeoff is a source of concern, particularly when the supposedly secondary task is as complex and demanding as random number generation. Consider, for example, the situation in which random number generation is combined with a simpler primary task that does not have much executive involvement. On the basis of a strategic tradeoff, one might expect severe decrements in primary task performance, but not necessarily in secondary task performance. In contrast, if random number generation is combined with a more complex, executive demanding primary task, performance on that primary task might be protected against performance decrement because of the strategic tradeoff, resulting in the impairment of performance of random number generation instead.

Note that a response selection bottleneck and a strategic tradeoff are inherently related factors. Tasks that are more complex and demanding tend to involve more steps and take more time to process. Therefore, they necessarily involve less frequent response selection.

These considerations suggest that when applied to the central executive, the simple dual-task logic might not always hold. More specifically, the amount of decrement in primary task performance may not necessarily reflect the extent of central executive involvement when the primary task requires rapid sequences of simple, easy judgments.

In this study, we asked the participants to perform three different psychometric visuospatial tests both alone and while concurrently performing a secondary task. The three tests we used, illustrated in Figure 1, were the paper folding, card rotations, and identical pictures tests (Ekstrom, French, Harman, \& Derman, 1976). According to factor-analytic studies (see, e.g., Carroll, 1993; Lohman, 1979), these tasks load on different spatial ability factorsspatial visualization, spatial relations, and visuospatial perceptual speed, respectively. Spatial visualization tasks (e.g., the paper folding test) require performance of a complex sequence of mental manipulations, whereas spatial relations tasks (e.g., the card rotations test) involve simple rapid manipulation of two-dimensional objects, usually within a single step (Carroll, 1993). Perceptual speed tasks (e.g., the identical pictures test) require no spatial transformations at all and primarily involve simple matching of visuospatial patterns (Carroll. 1993).

We chose these tasks because they appear to differ in terms of overall difficulty and demand on the central executive component, as well as in the extent to which rapid response selection is required. In terms of central executive demand, we propose that the paper folding test is the most demanding, because it requires a sequence of internal spatial transformations to be performed on the stimulus, which therefore requires management of taskspecific goals and subgoals as well as scheduling and coordination of different cognitive processes. The card rotations test should implicate the central executive to a lesser extent. Although this test also requires mental transformation of the stimulus, only a single spatial transformation (mental rotation) must be made for each item. The identical pictures test should require the least amount of central executive involvement, because it merely requires maintaining a visuospatial representation of a simple figure in memory to be matched against the answer choices ${ }^{1}$ and does not require any spatial transformation of the stimulus, extensive goal management, or planning. In sup- 
Paper Folding Test
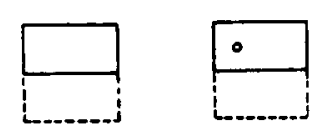

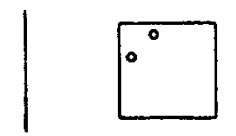

A

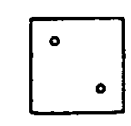

B

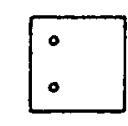

C

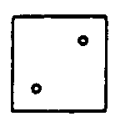

D

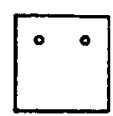

$\mathbf{E}$

\section{Card Rotations Test}

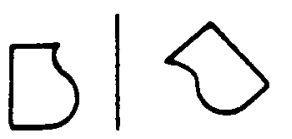

$\mathbf{A}$

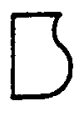

B

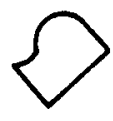

C

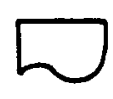

D

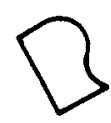

$\mathbf{E}$

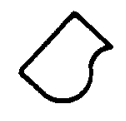

F

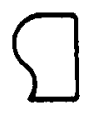

G

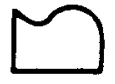

H

\section{Identical Pictures Test}

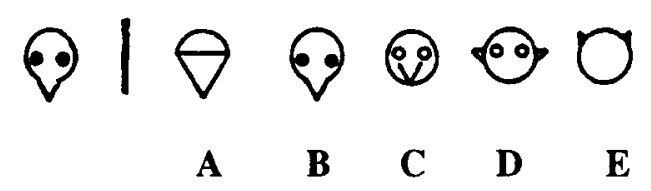

Figure 1. Typical trials in the three visuospatial psychometric tests.

port of this rank ordering, a recent individual differences study we conducted (Miyake, Rettinger, Friedman, Shah, \& Hegarty, 1999) showed that these three tasks involve different degrees of central executive functioning. A composite measure of central executive capacity, which consisted of participants' performance on the random number generation task and the Tower of Hanoi task (also considered a central executive task; Lehto, 1996; Shallice, 1988) correlated most highly with the paper folding test $[r(84)=$ $.42, p<.001]$, less with the card rotations test $[r(84)=.38$, $p<.001]$, and least with the identical pictures test $[r(84)=$ $.21, p<.10]$.

In contrast, in terms of the necessity to make rapid response selections, the rank ordering of the three psychometric tasks is exactly the opposite. The items in the identical pictures test are designed to be very simple, so that speed is the limiting factor in performance (Carroll, 1993). The card rotations test is also a simple speeded test (Lohman, 1979), although the mental transformation required for the performance of this task makes it more complicated than the identical pictures test. In contrast, an individual's performance on the paper folding test is primarily constrained by the difficulty of the items to be solved and, hence, the time limit is relatively liberal (Carroll, 1993). Indeed, completing all the items in these psychometric tests in the time allotted in our study required making 32.0 responses per minute in the identical pic- tures test, 13.3 responses per minute in the card rotations test, and 3.3 responses per minute in the paper folding test.

According to the simple version of the dual-task logic outlined above, concurrent performance of an executive task should interfere most with the paper folding test, less with the card rotations test, and least with the identical pictures test. However, if the two inherently correlated complicating factors we outlined above - a response selection bottleneck and a strategic tradeoff-play a role, the opposite pattern of interference should be observed, at least for the degree of performance decrement on the primary task.

In this study, we administered five different types of secondary tasks, two of which are considered to heavily involve the operations of the central executive component. These two tasks were random number generation and an auditory version of the 2-back task. The random number generation task, as discussed earlier, has been used to demonstrate the role of the central executive in cognitive tasks. The 2-back task involves searching for target letters in a continuous stream of letters. The participant is required to respond "yes" every time the letter is the same as the letter exactly two items before it and "no" otherwise. It has been argued that this task involves a variety of executive functions, including temporal tagging of incoming letters, updating and monitoring of actively main- 
tained information, and inhibition of responses to misleading foils (Cohen et al., 1997; Jonides \& Smith, 1997). We included this additional executive task to ensure that the results obtained in the study were not specific to random number generation.

In addition to the executive tasks, we administered three secondary tasks. One was the spatial tapping task, which is widely used to tap the visuospatial sketchpad component (particularly its spatial aspects) and, hence, should interfere with performance on all three visuospatial psychometric tasks. The other two secondary tasks were articulatory suppression, which specifically taps the phonological loop component, and simple tapping, which involves repeatedly pressing a single key and, hence, probably does not place much demand on working memory. Neither of these two secondary tasks should interfere with the performance of the three psychometric tests.

\section{METHOD}

\section{Participants}

One hundred thirty undergraduate students at the University of California, Santa Barbara, participated in the experiment for either course credit or a $\$ 10$ payment. Data from 10 participants were omitted from the analysis because of either equipment failure or experimenter error (e.g., the tasks were timed incorrectly), so the final analyses were based on data from 120 participants.

\section{Design}

The participants were randomly assigned to one of the five experimental groups; each group differed in the secondary task to be performed, which could be random number generation, 2-back, spatial tapping, articulatory suppression, or simple tapping ( $n=24$ for each group). Each participant performed the three visuospatial psychometric tests (paper folding, card rotations, and identical pictures) with and without a secondary task.

\section{Materials}

Primary tasks. The three visuospatial psychometric tests were selected from the Kit of Factor-Referenced Cognitive Tests (Ekstrom et al., 1976). All three tests included two equivalent subsections; the participants performed one subsection with a secondary task and the other subsection without a secondary task. A sample item for each test is presented in Figure 1.

In the paper folding test, the participants were required to mentally fold a piece of paper, imagine a hole punched through the folded paper, and then judge what the paper would look like when unfolded. The participants responded by choosing one of five alternatives. The items on this test got progressively more difficult, with the most complex items requiring three folds, which were sometimes nonsymmetrical. Each subsection included 10 items, and the time limit was $3 \mathrm{~min}$. In the card rotations test, the participants were required to view a two-dimensional target figure and indicate which of the test figures were planar rotations of the target figure (as opposed to its mirror image) as quickly and as accurately as possible. There were 10 rows of eight test figures in each subsection that required an average of 4 responses per row, and the time limit was $3 \mathrm{~min}$. In the identical pictures test, the participants were required to view a target figure and judge which one of the five alternative test figures was identical to the target figure as quickly and as accurately as possible. There were 48 items in each subsection, and the time limit was $1.5 \mathrm{~min}$.

We computed a score for each subsection of each of the three psychometric tests as follows: First, we counted the total number of correctly answered items. The number of incorrectly answered items was then divided by the number of alternatives for each item on the test and subtracted from the total number of correct answers (to correct for guessing). The highest possible score for each subsection of the psychometric tests was 10,80 , and 48 for the paper folding, card rotations, and identical pictures tests, respectively. Note that the maximum score for the card rotations test (i.e., 80 ) is twice the number of responses to be made (i.e., 40 ), because, in this test, credit was given for both indicating correct answers (i.e., hits) and not indicating incorrect answers (i.e., correct rejections; Ekstrom et al., 1976).

Secondary tasks. In the random number generation task, the participants were required to generate random numbers (between 0 and 9) at the rate of $1 / \mathrm{sec}$ to the beat of a metronome. The participants were instructed to say numbers in as random an order as possible and to try not to follow any particular number patterns while doing this task. We derived two different indices of the participants' performance on this task-degree of randomness of the produced numbers, and the rate of producing numbers. The degree of randomness was assessed in terms of an adjacency score (Towse \& Valentine, 1997). This measure reflects the percentage of two consecutive responses that are in numerical sequence (e.g., 3 followed by 4,7 followed by 6 ) relative to the total number of consecutive responses. ${ }^{2}$ The rate of producing numbers was the number of responses per second so that any values smaller than 1 would indicate that the participant had failed to produce the numbers to the beat of the metronome. To guard against the possibility of a speed-accuracy tradeoff, the two measures were combined by converting them to $z$ scores. However, the same general pattern of results was obtained for both measures when they were analyzed individually.

In the 2-back task, the participants were required to listen to a series of consonant letters that were played on a tape recorder at a speed of one every $2 \mathrm{sec}$. Their task was to respond by saying "yes" when the consonant that they heard last was identical to the consonant presented exactly two items before and to respond "no" to all other items. One third of the items were yes trials. In addition, one sixth of the trials were foils such that the consonant that was heard last was identical to the immediately previous consonant (one twelfth) or to the consonant exactly three items before (one twelfth). The percentage of correctly answered trials was used to assess performance on the 2-back task.

In the spatial tapping task, the participants were required to tap a square spatial pattern around a numerical keypad by tapping the numbers $1,4,7,8,9,6,3$, and 2 in order with the index finger of the dominant hand at the rate of 1 tap per second. To avoid the possibility that dual-task performance decrement was caused by inputlevel interference (i.e., the inability to look at the psychometric test booklets and the keypad at the same time), we instructed the participants to perform the assigned tapping task without looking at the keypad. In the articulatory suppression task, the participants were required to repeat the word the aloud every half second. Finally, in the simple tapping task, the participants were required to press a single key on the numeric keypad (1) at the rate of 1 tap per second. The responses were paced by a metronome for these three secondary tasks.

\section{Procedure}

The participants were first told that they would be performing three visuospatial paper-and-pencil tasks with and without a secondary task. They were then introduced to the secondary task that they would be performing and were allowed to practice it for $1 \mathrm{~min}$. Performance on the random number generation and 2-back tasks during this 1 -min practice period was used as a baseline measure to evaluate the degree of secondary task decrement in dual-task conditions.

The participants were then given the standard instructions for the first psychometric test, along with the standard practice items for that task. They performed the two subsections of that test, one with 
Table 1

Means, Standard Deviations, and $t$-Test Results Comparing Control and Dual-Task Performance on the Psychometric Tests in All Conditions

\begin{tabular}{|c|c|c|c|c|c|c|}
\hline \multirow[b]{2}{*}{ Condition } & \multicolumn{2}{|c|}{ Control } & \multicolumn{2}{|c|}{ Dual Task } & \multirow[b]{2}{*}{$t$} & \multirow[b]{2}{*}{$p$} \\
\hline & $M$ & $S D$ & $M$ & $S D$ & & \\
\hline \multicolumn{7}{|c|}{ Random Number Generation } \\
\hline Identical pictures & 36.65 & 4.72 & 25.41 & 7.54 & 8.26 & $<.016$ \\
\hline Card rotations & 56.79 & 17.50 & 44.00 & 17.49 & 4.40 & $<.016$ \\
\hline Paper folding & 5.21 & 2.15 & 4.01 & 2.72 & 2.90 & $<.016$ \\
\hline \multicolumn{7}{|l|}{ 2-back Task } \\
\hline Identical pictures & 38.55 & 7.13 & 26.83 & 7.14 & 7.13 & $<.016$ \\
\hline Card rotations & 61.90 & 16.08 & 48.35 & 15.81 & 5.21 & $<.016$ \\
\hline Paper folding & 4.82 & 1.94 & 3.65 & 2.42 & 2.32 & .03 \\
\hline \multicolumn{7}{|l|}{ Spatial Tapping } \\
\hline Identical pictures & 35.77 & 6.15 & 27.98 & 5.13 & 5.59 & $<.016$ \\
\hline Card rotations & 59.80 & 14.88 & 48.38 & 14.57 & 4.81 & $<.016$ \\
\hline Paper folding & 5.53 & 2.91 & 4.60 & 2.42 & 1.81 & .08 \\
\hline \multicolumn{7}{|c|}{ Articulatory Suppression } \\
\hline Identical pictures & 37.89 & 6.93 & 37.03 & 6.88 & 0.77 & n.s. \\
\hline Card rotations & 57.13 & 16.57 & 54.65 & 15.85 & 1.09 & n.s. \\
\hline Paper folding & 4.93 & 2.60 & 4.95 & 2.60 & -0.06 & n.s. \\
\hline \multicolumn{7}{|l|}{ Simple Tapping } \\
\hline Identical pictures & 39.71 & 6.24 & 37.27 & 6.38 & 2.26 & .03 \\
\hline Card rotations & 58.44 & 17.43 & 58.27 & 15.31 & 0.06 & n.s. \\
\hline Paper folding & 3.87 & 2.27 & 4.87 & 2.42 & -2.05 & .05 \\
\hline
\end{tabular}

the secondary task and the other without it. When a secondary task was performed, the participants started performing that task first, and as soon as they had begun, they were instructed to start the primary task. They stopped performing both tasks when all the items in that section of the primary task were completed or when the time limit for the primary task was reached, whichever came first. This procedure was repeated for each of the other two psychometric tests. We counterbalanced the order of the three psychometric tests, the order of the two subsections for each test, and the order of the dual-task and control conditions. ${ }^{3}$

The participants responded to the psychometric tests either by orally indicating an answer or by pointing to the answer choices on the test, depending on the response requirements of the secondary tasks. The participants whose secondary tasks required an oral response (i.e., in the random number generation, 2-back, and articulatory suppression tasks) responded by pointing to the answer choices. The participants whose secondary tasks required a tapping response (i.e., in the simple tapping and spatial tapping tasks) responded by saying the answer aloud. In the case of the card rotations test, responding to each test item involved indicating each answer choice that was a planar rotation of the target figure (among the eight answer choices presented). The number of correct answer choices varied from trial to trial with an average of four. In the case of the identical pictures and paper folding tests, responding to each test item involved indicating one answer choice among the five alternatives presented.

\section{RESULTS AND DISCUSSION}

\section{Impairment of Psychometric Task Performance by the Secondary Tasks}

The main purpose of this study was to examine the extent to which concurrent performance of the two secondary executive tasks (i.e., the random number generation and 2-back tasks) would disrupt participants' performance on the three different psychometric tests. To this end, we first examined whether each of the five secondary tasks would significantly impair performance on each of the visuospatial psychometric tests. Table 1 summarizes the results of this analysis, indicating the means, standard deviations, and $t$ test results from comparing the scores of each psychometric task performed with and without the secondary task requirement. Because three $t$ tests were performed for each secondary task, we set the alpha level at .016 for the results reported in Table 1, using the DunnBonferroni procedure for multiple a priori comparisons (Winer, Brown, \& Michaels, 1991).

As Table 1 indicates, random number generation impaired performance on all three psychometric tests. The other executive task, the 2-back task, had a significant dual-task effect on the identical pictures and card rotations tests, but only a marginally significant effect on the paper folding test. Spatial tapping had the same pattern of results as the 2-back task, showing a significant effect on the identical pictures and card rotations tests, but not on the paper folding test, although there was a marginally significant trend in the predicted direction. As expected, there were no reliable dual-task effects of articulatory suppression on any of the psychometric tests, suggesting that these psychometric tasks do not depend on the phonological loop. Simple tapping also showed no statistically significant dual-task effect on the three psychometric tests, although simultaneous performance of this task led to a marginally significant impairment on the identical pictures test. A marginally significant facilitation effect of simple tapping on the paper folding test probably reflects the unusually low performance on this psychometric test in the control condition for the participants in the simple tapping condition. 


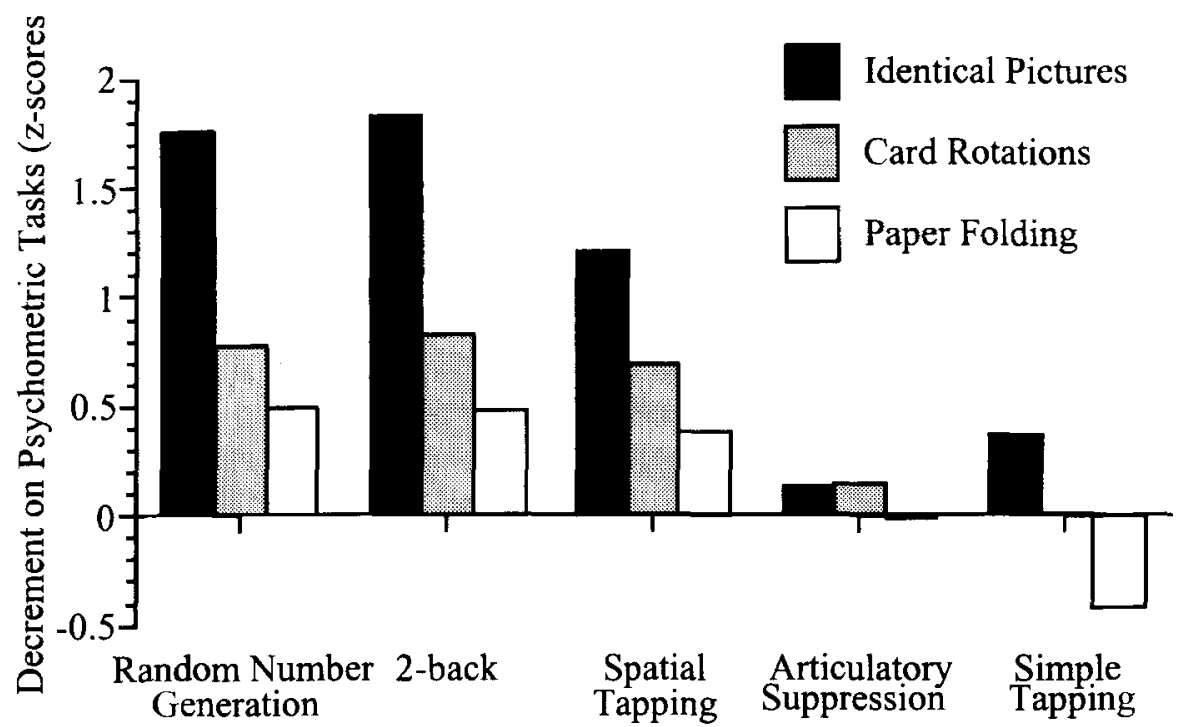

Figure 2. Mean decrement on the three psychometric tests for each secondary task group. The decrement scores express the difference between the psychometric test scores performed under the control and the dual-task conditions in terms of the standard deviation of the control condition.

\section{Relative Amounts of Interference by Different Secondary Tasks}

The two executive tasks. The crucial analysis compares the relative interference effects of the two executive tasks (the random number generation and 2-back tasks) on the three psychometric tests. For this analysis, we computed a standardized measure of performance decrement due to each secondary task on each of the psychometric tests. More specifically, we subtracted the score of the psychometric task in the dual-task condition from the score of the same task in the control condition and divided that difference by the standard deviation of the task in the control condition. Thus, the values plotted in Figure 2 express the degree of decrement in performance in the dual-task conditions in terms of $z$ scores.

As Figure 2 shows, the two secondary executive tasksrandom number generation and 2-back--showed a remarkably similar pattern. Contrary to the prediction based on the simple dual-task logic, the identical pictures test (the simplest psychometric task of the three) showed the largest performance decrement, whereas the paper folding test (the most complex and demanding psychometric test) showed the least decrement. A one-way within-subjects analysis of variance confirmed this observation, indicating that the degree of performance decrement due to the concurrent secondary task was different for the three psychometric tests both when the secondary task was random number generation $\left[F(2,46)=12.62, M S_{\mathrm{e}}=0.83, p<\right.$ $.01]$ and when it was the 2-back task $[F(2,46)=12.23$, $\left.M S_{\mathrm{e}}=0.96, p<.01\right]$. Post hoc analyses indicated that, for both secondary tasks, the degree of decrement for the identical pictures test was statistically greater than that for the card rotations and paper folding tests $(p<.05)$, which did not significantly differ from each other $(p>.10)$.

This rank ordering of the magnitude of interference effects is clearly inconsistent with predictions from the simple dual-task logic, suggesting that other complicating factors must be taken into account. The two complicating factors that we proposed earlier, the response bottleneck factor and the strategic tradeoff factor, both predict the rank ordering of the three psychometric tests observed here. Indeed, further analyses of the data provided evidence that each of these factors might have played a role in yielding this result.

First, the contribution of the response selection factor was suggested by the following analysis: When the performance decrement was adjusted for frequency of response selection, there was no significant difference between the decrements on the three psychometric tests. To adjust for response selection frequency, we divided the $z$ score for each participant by the number of responses per minute made by that participant in the dual-task condition. For the random number generation condition, the adjusted decrement was .15 for the paper folding test, .08 for the card rotations test, and .11 for the identical pictures test $\left[F(2,46)=1.06, M S_{\mathrm{e}}=0.028, p>10\right]$. For the 2-back condition, the adjusted decrements were $.14, .09$, and .11 for the paper folding, card rotations, and identical pictures tests, respectively $\left[F(2,46)=0.42, M S_{\mathrm{e}}=0.037, p>10\right]$. Although this is admittedly a crude way of assessing the contribution of the response bottleneck factor, the results are nonetheless consistent with the view that the requirement to select responses rapidly in the two executive tasks did severely interfere with a similar response selec- 

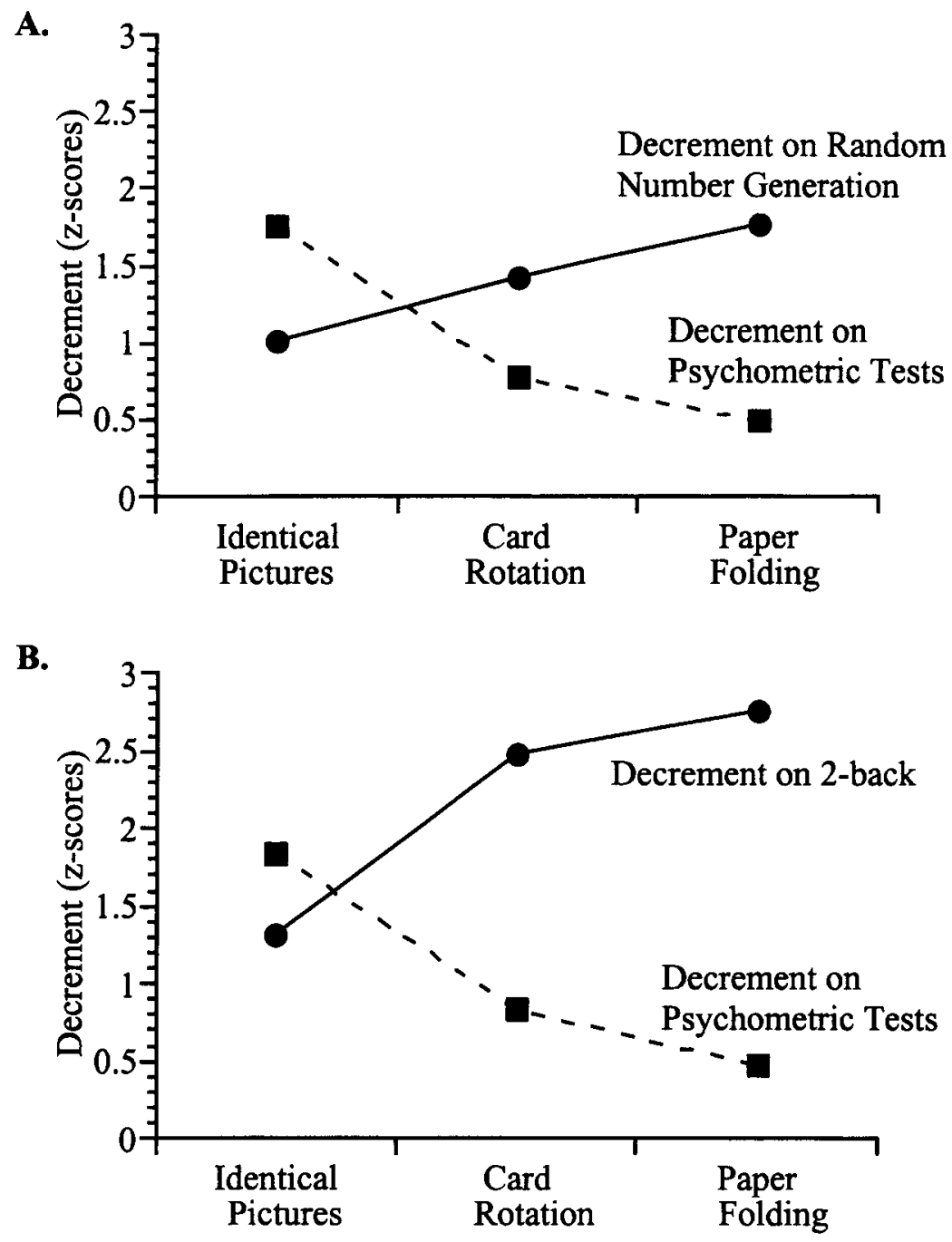

Figure 3. Mean decrement on the primary psychometric tests and secondary executive tasks for the random number generation group (A) and the 2-back group (B). For both primary and secondary tasks in each graph, the decrement scores express the difference between dual-task performance and control performance in terms of the standard deviation of performance in the control condition.

tion requirement that was associated with the identical pictures test.

Second, there was evidence for the involvement of a strategic tradeoff between the primary and secondary tasks. Consistent with a strategic tradeoff account, the decrement in secondary task performance (as indicated by the solid lines in Figure 3) showed the opposite pattern to that of the primary tasks (shown by the dashed lines). It was largest for the paper folding test and smallest for the identical pictures test for both the random number generation task (Figure 3A) and the 2-back task (Figure 3B). Although the degree of secondary task impairment on the random number generation task did not differ significantly among the three psychometric tests $[F(2,40)=$ $\left.2.40, M S_{\mathrm{e}}=1.56, p>.10\right]$, the trend was clearly in the direction suggested by the strategic-tradeoff account. ${ }^{4}$ For the 2-back task, the difference in the degree of secondary task performance impairment among the three psychometric tests was statistically significant $[F(2,46)=14.57$, $\left.M S_{\mathrm{e}}=0.98, p<.01\right]$. Post hoc tests indicated that the decrement for both the paper folding test and the card rotation test was greater than that for the identical pictures test ( $p<.001$ in both cases). These results strongly suggest that the rank ordering of performance decrements for the primary task that we documented earlier reflects, at least in part, the participants' strategic tradeoff between the two concurrent tasks.

Taken together, the results reported in this section challenge a simple version of the dual-task logic and highlight the necessity to take into consideration factors such as response selection requirements and strategic tradeoffs between primary and secondary tasks. 
Other secondary tasks. Interestingly, spatial tapping, a secondary task widely used to tap the visuospatial sketchpad component of working memory, also showed a pattern similar to that found for the two secondary executive tasks. As can be seen in Figure 2, concurrent performance of spatial tapping led to significantly different degrees of decrement for the three psychometric tasks $\left[F(2,46)=4.63, M S_{\mathrm{e}}=0.92, p<.05\right]$. Post hoc comparisons indicated that this was due to a significantly greater decrement on the identical pictures test than on the paper folding test $(p<.01)$, whereas the decrements between the other pairs of tests did not differ significantly. This pattern of results was somewhat unexpected, but, like the random number generation task and the 2-back task, spatial tapping may involve a sequence of rapidly selected responses. To the extent that this might be the case, the overall pattern of results for this task may have been a consequence of a response selection bottleneck.

The other two secondary tasks, articulatory suppression and simple tapping, did not have as large a negative impact on the performance of the three psychometric tests (particularly, articulatory suppression). Although the rank ordering of the tasks for the simple tapping condition was the same as that for the two executive tasks and the spatial tapping task, the pattern was qualitatively different in that there was virtually no performance decrement for the card rotations test and there was some facilitation for the paper folding test.

\section{GENERAL DISCUSSION}

The main result of the experiment can be summarized as follows: Contrary to the prediction based on the simple dual-task logic, the decrement in the participants' primary task performance when paired with secondary executive tasks was smallest for the task considered to most heavily involve the central executive (i.e., the paper folding test) and was largest for the task considered to be the least demanding of executive mechanisms (i.e., the identical pictures test). This result suggests that, when applied to the assessment of central executive involvement, the prevalent simple dual-task logic does not always apply and needs to be used cautiously when the secondary task is as complex and demanding as the random number generation and 2-back tasks.

Earlier, we suggested two complicating factors that may limit the application of the dual-task logic. The first concerned the situation in which the primary task required a series of rapidly selected responses. Because the executive tasks typically used as secondary tasks (e.g., random number generation) also involve rapid response selection, the primary task may suffer from a severe interference effect, even if the degree of central executive involvement for that task is low. The contribution of this factor has not received much attention in the dual-task studies of central executive involvement. This is, perhaps, because published studies have focused primarily on highly com- plex tasks (e.g., chess playing and syllogistic reasoning) that are similar to the paper folding test (the most complex task considered here) in terms of the frequency of response selections.

The second complicating factor concerned strategic tradeoffs. If the primary task is much less demanding than the designated secondary executive task, participants might allocate more effort or attention to the secondary task to protect their performance on that task against a major decrement (Bourke et al., 1996; Navon \& Gopher, 1979). This strategic tradeoff can cause a deterioration in performance of the easy primary task, even if it does not necessarily place much demand on the central executive. This factor seems to be better recognized in the dual-task literature than is the response selection factor, but not all dual-task studies published so far report the degree of secondary task performance decrements. Our data suggest that it is essential to report the dual-task decrement not just for primary tasks but also for secondary tasks, particularly when relatively complex secondary tasks are used.

Taken together, the overall results reported in this study provide important constraints on the usage of dual-task methodology to assess the degree of central executive involvement in cognitive tasks. These results strongly suggest that, when the presence of two complicating factors (i.e., response selection and strategic tradeoffs) is suspected, one must either carefully control for these factors or use other methods (e.g., correlational analyses) in order to assess the degree of central executive involvement.

These qualifications of the dual-task logic are applicable primarily to investigation of central executive functioning through the use of demanding executive tasks as secondary tasks. The simple logic may be less susceptible to these constraints when used for examining phonological loop involvement through the method of articulatory suppression, because simply repeating the same familiar word or phrase is an automatized, nondemanding task and involves minimal response selection. Some secondary tasks used to tap the visuospatial sketchpad component, such as viewing irrelevant pictures (Quinn \& McConnell, 1996), are probably less susceptible to these constraints as well. As our data indicate, however, the spatial tapping technique may require some response selection processes and, hence, should be used cautiously when the primary task also involves rapid response selection.

One possible objection to these conclusions is that there is nothing wrong with the simple dual-task logic even when it is applied to central executive involvement. Instead, the reason that executive tasks interfere with the identical pictures test is that response selection is an important function of the central executive and, hence, the identical pictures test involves central executive functioning. We acknowledge this possibility, but would like to point out some problems. One problem with this argument is that it is essentially impossible to think of any sufficiently complex cognitive task that would not show any 
significant performance decrement when concurrently performed with demanding executive tasks. If executive tasks, such as random number generation and 2-back, impair virtually all cognitive tasks, it is uninformative to examine the involvement of the central executive by using them as secondary tasks. Another problem with the argument that the identical pictures test is an executive task is that it fails to explain several other results. First, it fails to explain why executive tasks have higher correlations with the paper folding test than with the identical pictures test (Miyake et al., 1999). ${ }^{5}$ Second, it fails to explain why the performance decrement for the primary task is disproportionately larger for the identical pictures test than for the paper folding test, or why this ordering reverses when the performance decrement for the secondary task is assessed.

One possible way to address these problems and still maintain the simple dual-task logic is to argue that the central executive is not a unitary construct but instead consists of different subcomponents or subprocesses. According to this account, the degree of performance decrement in dual-task situations depends on the extent to which the primary and secondary tasks involve the same subcomponents or subprocesses of the nonunitary central executive (Baddeley, 1996; Monsell, 1996; Shallice \& Burgess, 1996). This idea is worthy of further investigation; however, such an approach would involve identifying specific subcomponents or subprocesses of the central executive, devising tasks that involve one specific executive function (but not others), and using these tasks as secondary tasks in dual-task situations, rather than solely relying on random number generation or the 2-back test as secondary executive tasks. This approach has recently been used by Baddeley and his colleagues to specify the involvement of one often postulated executive function (i.e., attention switching) in the performance of random number generation (Baddeley, Emslie, Kolodny, \& Duncan, 1998).

In conclusion, the results of the present study help sharpen the logic behind dual-task experiments when they are applied to the evaluation of the degree of central executive involvement, and they provide useful constraints on the types of so-called executive tasks that may or may not be used as secondary tasks for that purpose, depending on the nature of the primary task of interest. Despite such constraints, the dual-task methodology can still be a useful and informative experimental paradigm when used appropriately and can be a benefit to our understanding of working memory and its role in human cognition.

\section{REFERENCES}

BADDELEY, A. D. (1966). The capacity for generating information by randomization. Quarterly Journal of Experimental Psychology, 18, 119-130.

BADDEley, A. D. (1992). Working memory. New York: Oxford University Press.

BadDeley, A. D. (1996). Exploring the central executive. Quarterly Journal of Experimental Psychology, 49A, 5-28.
Baddeley, A. D., Emslie, H., Kolodny, J., \& DunCan, J. (1998). Random generation and the executive control of working memory. Quarterly Journal of Experimental Psychology, 51 A, 819-852.

BadDeley, A. D., \& Hitch, G. (1974). Working memory. In G. H. Bower (Ed.), The psychology of learning and motivation (Vol. 8, pp. 47-89). New York: Academic Press.

BADDElEY, A. D., \& Logie, R. H. (1999). Working memory: The multiple component model. In A. Miyake \& P. Shah (Eds.), Models of working memory: Mechanisms of active maintenance and executive control (pp. 28-61). New York: Cambridge University Press.

Bourke, P. A., DunCan, J., \& Nimmo-Smith, I. (1996). A general factor involved in dual-task performance decrement. Quarterly Journal of Experimental Psychology, 49A, 525-545.

CARroll, J. (1993). Human cognitive abilities: A survey of factoranalytic studies. New York: Cambridge University Press.

Cohen, J. D., Perlstein, W. M., Braver, T. S., Nystrom, L. E., Noll, D. C., Jonides, J., \& SMith, E. E. (1997). Temporal dynamics of brain activation during a working memory task. Nature, 386, 604608.

Ekstrom, R. B., French, J. W., Harman, H. H., \& Derman, D. (1976). Kit of factor-referenced cognitive tests. Princeton, NJ: Educational Testing Service.

GatherCole, S. E., \& BadDeley, A. D. (1993). Working memory and language. Hillsdale, $\mathrm{NJ}$ : Erlbaum.

GilhoOLY, K. J., LOGIE, R. H., WeTHERICK, N. E., \& WYNN, V. (1993). Working memory and strategies in syllogistic-reasoning tasks. Memory \& Cognition, 21, 115-124.

JoNIDES, J., \& SMITH, E. E. (1997). The architecture of working memory. In M. D. Rugg (Ed.), Cognitive neuroscience (pp. 243-276). Cambridge, MA: MIT Press.

LEHTO, J. (1996). Are executive function tests dependent on working memory? Quarterly Journal of Experimental Psychology, 49A, 2950.

LOGIE, R. H. (1995). Visuo-spatial working memory. Hove, U.K.: Erlbaum.

LoGIE, R. H., GiLhooly, K. J., \& WYNN, V. (1994). Counting on working memory in arithmetic problem solving. Memory \& Cognition, 22, 395-410.

Lohman, D. (1979). Spatial ability: Individual differences in speed and level (Tech. Rep. No. 9). Stanford, CA: Stanford University, School of Education.

Miyake, A., Rettinger, D., Friedman, N. P., Shah, P., \& Hegarty, M. (1999). Reinterpreting psychometric spatial abilities with a multicomponent model of working memory. Manuscript submitted for publication.

MonselL, S. (1996). Control of mental processes. In V. Bruce (Ed.), Unsolved mysteries of the mind: Tutorial essays in cognition (pp. 93148). Hove, U.K.: Psychology Press.

NAvON, D., \& Gopher, D. (1979). On the economy of the humanprocessing system. Psychological Review, 86, 214-255.

PASHLER, H. (1994). Dual-task interference in simple tasks: Data and theory. Psychological Bulletin, 116, 220-244.

QuinN, J. G., \& MCConNell, J. (1996). Irrelevant pictures in visual working memory. Quarterly Journal of Experimental Psychology, 49A, 200-215.

Robbins, T. W., Anderson, E. J., Barker, D. R., Bradley, A. C., Fearneyhough, C., Henson, R., Hudson, S. R., \& Baddeley, A. D. (1996). Working memory in chess. Memory \& Cognition, 24, 83-93.

Salway, A. F. S., \& Logie, R. H. (1995). Visuospatial working memory, movement control and executive demands. British Journal of Psychology, 86, 253-269.

SHALliCE, T. (1988). From neuropsychology to mental structure. New York: Cambridge University Press.

Shallice, T., \& Burgess, P. (1996). The domain of supervisory processes and temporal organization of behaviour. Philosophical Transactions of the Royal Society of London: Series B, 351, 1405-1411.

TOWSE, J. N. (1998). On random generation and the central executive of working memory. British Journal of Psychology, 89, 77-101. 
Towse, J. N., \& VAlEntine, J. D. (1997). Random generation of numbers: A search for underlying processes. European Journal of Cognitive Psychology, 9, 381-400

Winer, B. J., Brown, D. R., \& Michaels, K. M. (1991). Statistical procedures in experimental design (3rd ed.). New York: McGraw-Hill.

\section{NOTES}

1. As the sample item in Figure 1 shows, this test involved viewing the target figure on the left and matching it against the answer choices on the right. In some cases, the target and the correct answer might be viewed in a single eye fixation (e.g., when the correct item was answer choice A), but in most cases, this test required the maintenance of the target item across fixations. Thus, some form of temporary storage of visuospatial information was required in this task.

2. Although it is rather crude, we chose the adjacency measure of degree of randomness in this study for a number of reasons. First, it has been shown to be sensitive to dual-task demands (Towse \& Valentine, 1997). Second, other more sophisticated measures (see, e.g., Baddeley, Emslie, Kolodny, \& Duncan, 1998; Towse, 1998) typically require a large number of responses for a reliable assessment of degree of ran- domness. In this study, the control (i.e., single-task) condition lasted only $1 \mathrm{~min}$ and, hence, the participants generated a maximum of 60 numbers, which put constraints on our selection of the randomness measure.

3. We collapsed over task order (i.e., control condition first vs. dualtask condition first) because we completely counterbalanced this variable. Additional analyses showed, however, that the main effect of task order was nonsignificant in all of the conditions.

4. Because of equipment failures, the analyses of secondary task performance for the random number generation task are based on the data from 21 of out of 24 participants.

5 . It could be argued that the identical pictures test does not correlate highly with executive tasks because the executive processes implicated in the task are not the main limiting factor for a participant's level of performance. This argument might explain why executive tasks impair performance most in the identical pictures test in dual-task situations, but does not explain why the order of impairment reverses when the performance decrement for the secondary task is assessed.

(Manuscript received June 30, 1998; revision accepted for publication May 11, 1999.) 